
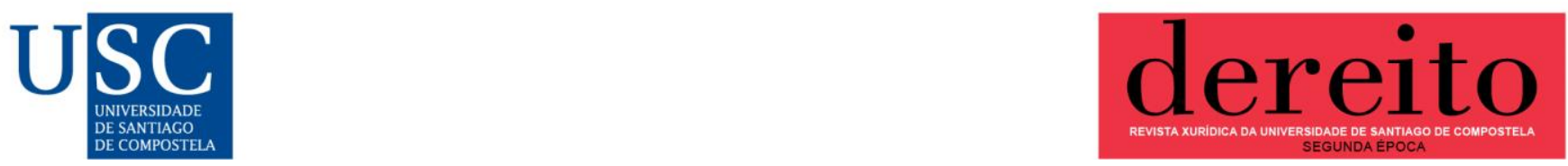

Dereito: revista xurídica da Universidade de Santiago de Compostela, 30 (1), 2021. ISSN: 2174-0690

https://doi.org/10.15304/dereito.30.1.7388

Notas

\title{
Docencia, derecho, ópera. Prima la musica, poi le parole? ${ }^{1}$
}

Teaching, law, opera. Prima la musica, poi le parole?

José María Miranda Boto

Universidad de Santiago de Compostela, España

josemaria.miranda@usc.es

\section{Resumen}

La Universidad debe ser un vehículo de formación de ciudadanos, en los que se instilen intereses culturales junto con conocimientos técnicos. El texto reflexiona sobre el encaje de la ópera en los estudios de Derecho, proponiendo la utilización de los contenidos musicales como notas al pie de las enseñanzas. Se buscará con ella suscitar la curiosidad del estudiante, que le lleve a descubrir con este estímulo el "arte definitivo". El artículo propone un elenco de conexiones jurídico-musicales, que recorren la historia de la ópera y una buena extensión del ordenamiento jurídico, de Mozart a Berg, del Derecho Penal al Derecho del Trabajo.

Palabras clave: Docencia, Derecho, Ópera, Violencia de Género

\begin{abstract}
The University must be a vehicle for the formation of citizens, in which cultural interests are instilled along with technical knowledge. The text reflects on how opera fits into Law studies, proposing the use of musical content as a footnote to the teaching. The aim is to arouse the curiosity of the student, leading him or her to discover the "definitive art" with this stimulus. The article proposes a list of legal-musical connections.
\end{abstract}

"El profesor tiene que despertar deseos, aunque no pueda satisfacerlos. Deseo de saber, sin duda; más aún, deseo de ver, de mirar, de preguntarse, de quedarse perplejo, de moverse en un mundo mágico, que el joven casi siempre desconoce y que el profesor descubre, entreabriendo una puerta, quizá sin atreverse a franquearla él mismo".

Julián Marías, “El profesor universitario” (1983) 


\section{1. ¿CULTURA EN LAS ENSEÑANZAS JURÍDICAS?}

Quien esto firma tiene como dogma personal que la Universidad no es un lugar consagrado a la formación de técnicos, sino a la creación de ciudadanos, responsables y con intereses humanísticos. No cabe duda de que esta es una opinión que no va con el Zeitgeist. Basta con evocar el informe de la Autoridad Independiente de Responsabilidad Fiscal sobre el sistema universitario andaluz $^{2}$ para ver que no son buenos tiempos ni para la lírica ni para este tipo de saberes extraprofesionales.

No obstante, la causa de la Cultura, con mayúscula, ha de ser defendida. ¿Qué sentido tiene entrenar técnicos que permanezcan impávidos ante los grandes logros de la Historia del Arte? ¿Han de abandonar las Universidades nuestros estudiantes sin sentir el menor interés por la civilización en la que viven? ¿Serán repertorios de jurisprudencia disecados? Es obligado que los profesores, si nos sentimos parte de esa civilización, aportemos algo a esa construcción de juristas de mundo y no de legajo.

Es cierto que la noción misma de "Cultura" es ambigua 3 . Las dos definiciones más relevante que proporciona la Real Academia Española son orientativas, pero no precisas: "2. f. Conjunto de conocimientos que permite a alguien desarrollar su juicio crítico. 3. f. Conjunto de modos de vida y costumbres, conocimientos y grado de desarrollo artístico, científico, industrial, en una época, grupo social, etc". No en vano, algunas de las mentes más preclaras ${ }^{4}$ de la Historia han dedicado páginas y páginas a su caracterización. Sirva para este trabajo la grandilocuente caracterización que propuso de "Alta Cultura" el británico Matthew Arnold: "know the best that has been said and thought in the world"5.

Los estudiantes de Derecho deberían percibir, pues, que hay puertas que les pueden conducir a un patrimonio inmaterial, del que podrán apropiarse gozosos.Una primera vía para ello, que no voy a abordar, la integra la enseñanza de un "Derecho del Arte" o "Derecho de la Cultura", donde podrían ensamblarse contenidos de diversas disciplinas. Su aspecto de asignatura optativa de conocimiento avanzado, incluyendo, naturalmente, amplias dosis de propiedad intelectual, no parece sin embargo el más apropiado para una difusión cultural, sino técnica. Esta aproximación puede realizarse también de forma sectorial, naturalmente. El epígrafe II del Tema 10 del programa de la asignatura "Derecho del Trabajo I" en el Grado en Derecho en la USC cubre, en el ámbito que me ocupa, de forma escueta este punto al abordar los derechos de propiedad intelectual de los trabajadores asalariados.

La segunda vía, cultivada también desde hace tiempo, es la utilización de textos literarios para el estudio de cuestiones jurídicas, principalmente relacionadas con el tema histórico o introductorio de la materia. En el campo del Derecho del Trabajo es habitual, para abordar la Cuestión Social del siglo XIX, textos de Benito Pérez Galdós (Marianela ${ }^{6}$ ), de Emilia Pardo Bazán ( $L a$ Tribuna7), o de Concepción Arenal (Cartas a un obrero $^{8}$ ), especialmente en el año de su bicentenario. Otras disciplinas tienen acceso a materiales que pueden resultar más atractivos, por su modernidad, a los estudiantes. Se busca así suscitar una curiosidad por textos que, en muchos casos, son pasados por alto o fueron objeto de examen en etapas formativas previas y del consiguiente posterior aborrecimiento. 
Mi personal aportación en este campo consiste en la utilización del Prólogo y los Capítulos "Una reunión muy esperada" y "La sombra del pasado", de El Señor de los Anillos, de J.R.R. Tolkien para introducir las notas características de la relación laboral ${ }^{9}$. Al utilizar a Frodo Bolsón y Samsagaz Gamyi para hacer discurrir a los alumnos sobre dichos rasgos en una historia literaria, no hago sino seguir el camino abierto por el Maestro Alonso Olea en su obra seminal "Entre Don Quijote y Sancho, ¿relación laboral”10. Llevo, eso sí, la discusión al campo del "Autor del Siglo XX”11.

La tercera vía, la que voy a defender en este trabajo, es la introducción de contenidos culturales en la docencia ordinaria. Probablemente, la forma más tradicional de poner en práctica esta idea es la utilización de nombres literarios en los casos prácticos, arrojando sugerentes migas de pan ante los alumnos dispuestos a seguirlas. Cuando yo estudiaba Derecho, el siglo pasado, mi joven profesor de Derecho Administrativo ya deslizaba nombres de la literatura de Juan Benet en los casos sobre expropiación y mi profesor de Instituciones de Derecho Comunitario no dejaba pasar una clase sin abrirnos al mundo de su erudición inagotable. Quizá fue entonces la primera vez que oí hablar con naturalidad de Borges.

De la misma manera, el tradicional caso práctico resulta enriquecido si en lugar de hablar de "la trabajadora" se le asigna como nombre "Ana Ozores" o si el empresario innominado asume las facciones de Antonio Cánovas del Castillo. Por supuesto, no todo ha de ser decimonónico, sino que cada docente puede incorporar a su galería personal a sus propios personajes de referencia, ya sea Jesús Quintana o Natalia Romanova. Pero tampoco hace falta exagerar.

Otra técnica para la introducción de "huevos de Pascua" es la utilización de cifras en clave. A la hora de indicar el número de trabajadores que tiene un centro de trabajo para determinar la composición de su comité de empresa, la elección de la cifra puede ser muy relevante. Si se utiliza 144 , se abre con ello el camino a mencionar las doce docenas que componen una gruesa entre los Hobbits. Si se utiliza 451, la referencia es a la temperatura a la que arde el papel en grados Fahrenheit, trayendo con ello la obra de Ray Bradbury y la película de François Truffaut. El CIF de la empresa, los códigos de cuenta de cotización o el salario de un trabajador son ocasiones para este acercamiento.

Una última propuesta a estos efectos es la de preparar los casos prácticos deslizando frases extraídas de obras literarias. Todos estos elementos pueden ser la madriguera de conejo que permita acceder a un mundo distinto del jurídico. La lúgubre redacción de un caso oscuro en el que se parodie la escritura de H.P. Lovecraft, por ejemplo, se puede llevar a la práctica. Pero no se puede olvidar que es una verdad universalmente reconocida que todo estudiante universitario necesita siempre puntos extra. Incitarles con una pequeña recompensa, gamificando así el sistema, no parece esfuerzo inútil. Ahora bien, mayor es la recompensa emocional del estudiante que reconoce la mención. Porque conocer, en el fondo, es reconocer.

La generalización de las aulas virtuales y de los contenidos online lleva este escenario a la enésima potencia y permite introducir la Ópera en este campo. En este momento, es posible adornar culturalmente casos prácticos, powerpoints, repositorios virtuales. Youtube, sin necesidad de excesivo conocimiento técnico, pone a disposición del docente una serie de recursos visuales que permite así introducir al "arte definitivo"12, la ópera, en las aulas. Es menester aprovechar esta marea en pleamar para que nos conduzca a la fortuna de traerla ópera a la Facultad de Derecho ${ }^{13}$. 


\section{2. ÓPERA Y DERECHO}

No hay Prólogo en la Historia de la Música que resista comparación con la declaración de intenciones de Ruggiero Leoncavallo en Pagliacci. Esta ópera, que es puro Derecho Penal y que ha sido objeto de minuciosos análisis jurídicos, se abre con lo que se ha considerado el manifiesto fundacional del Verismo. En las líneas que a cortina bajada el Prólogo canta, este nos cuenta, apartándose de la tradición de la Commedia dell'Arte, sus propósitos. Ahora he de hacerlo yo con los míos.

A la hora de valorar lo que he hecho con el tiempo que se me ha dado, ostento con orgullo la condición de Director de tres ciclos de Extensión Universitaria titulados "Ópera na Facultade", "Mais Ópera na Facultade" y "Ópera na Universidade", impartidos respectivamente en los cursos 2009-2010, 2010-2011 y 2014-2015. Con el señuelo de un crédito de libre configuración y la colaboración de personas del ámbito operístico y del ámbito jurídico, varias docenas de estudiantes, más de una gruesa, vieron por primera vez una ópera, siquiera en forma enlatada. Como colofón del curso de verano que dirigí con el título "Dereito en serio e de broma”, en el curso 2008-2009, proyecté, de forma comentada, el Gianni Schicchi de Giacomo Puccini, quizá la más jurídica de todas las óperas, como luego indicaré.

Llevo más de una década, pues, intentando extender a mis estudiantes el placer de esa forma sublime de arte a la que yo accedí, precisamente, cuando tenía su misma condición, allá por el curso 1995-1996. La fórmula, creo, ha de ser invertir la pregunta que, remedando el título de la ópera de Antonio Salieri de 1786, enhebra el Capriccio de Richard Strauss ${ }^{14}$. Las palabras han de ser la vía a través de la cual la ópera puede tener acceso a las aulas de la Facultad de Derecho. La trama traerá la excusa para la belleza de la música. Por lo tanto, prima le parole, poi la musica.

La selección ha de ser cuidadosa. Wozzeck, de Alban Berg, puede ser una fuente indudable de reflexión jurídica. ¿Es correcto el contrato que el desgraciado protagonista celebra con el innominado Doktor para servir como cobaya humana para sus enloquecidos experimentos ${ }^{15}$ ? ¿Cuántos delitos se cometen y qué formas de participación en ellos se representan? El problema es el shock del neófito que escuche por primera vez la partitura de Berg, que debe ser evitado. El shock, no Berg necesariamente.

Haciendo bueno el consejo de la corte musical de José II en Amadeus, será apropiado situar el escenario en la ópera italiana, al menos para empezar, y otros repertorios podrán ir siendo facilitados en pequeñas dosis. En más de cuatrocientos años de existencia sobre los escenarios, desde la Euridice de Iacopo Peri, el ramillete de ejemplos que se puede recoger es excelente.

A mi juicio, pocas óperas hay más jurídicas que Gianni Schicchi, la tercera hoja del Trittico de Puccini. Todo el argumento se construye sobre un fraude testamentario, objeto de los reproches de Dante en el Canto XXX del Infierno ${ }^{16}$. Una noble familia codiciosa, un plebeyo más listo que todos ellos juntos y una historia de amor añadida por Puccini y su libretista para endulzar un poco semejante dosis de malicia. Para el público general, solo existe esta ópera por $O$ mio babbino caro. 
Para el jurista, no existe, en cambio, fragmento de un libreto que pueda superar el "In Dei nomini, anno Dei nostri Jesu Christi, ab eius salutifera incarnatione millesimo ducentesimo nonagesimo nono, die prima septembris, indictione undecima, ego notaro Amantio di Nicolao, civis Florentiae, per voluntatem Buosi Donati scribo hoc testamentum... Annullans, revocans, et irritans omne aliud testamentum!" 17. No hay improvisación alguna en estas líneas, sino una reproducción minuciosa de los formulismos legales al uso. Tanto es así, que el testamento del pintor Piero della Francesca, datado en 1487 pero publicado en $1884^{18}$, sirve de perfecta guía para la ficticia última voluntad de Buoso Donati.

El inicio de la clase correspondiente de la materia Derecho de sucesiones puede ser el momento idóneo para proyectar este fragmento, no más de cinco minutos. En él se va a encontrar la base para presentar por primera vez las formalidades del testamento y los artículos 662 y siguientes del Código Civil. Dado que Schicchi finge ser el difunto Buoso Donati para dictar sus últimas voluntades, la nulidad recogida en el artículo 673 es una parada obligatoria.

Messer Amanzio de Nicolao permite traer a colación el personaje del Notario, omnipresente en el género y en el repertorio de Piero di Palma. Aunque en la obra comentada su función sea la de redactar un testamento, la tarea principal del fedatario público en la ópera italiana es la de ser testigo cualificado del matrimonio ${ }^{19}$. Ante la imposibilidad de representar la celebración de un sacramento en escena, tal era la fórmula escogida por los libretistas para representar el casamiento, algo muy habitual en el género, tanto como el crimen. Normalmente, por cierto, el compromiso matrimonial se viene abajo en el acto siguiente, como en L'elisir d'amore, o el notario no es más que un personaje disfrazado, como sucede en la travestida Despina de Così fan tutte, ópera digna de figurar en los anales de la misoginia. Derecho Eclesiástico es, sin duda, el escenario adecuado para suscitar uno de estos fragmentos.

Otros actos jurídicos tienen lugar en otras óperas, desde el enrolamiento de Nemorino en L'elisir d'amore a los vasos de vino de Turiddu en Cavalleria rusticana. Las hipócritas menciones a la Ley por parte del barón Scarpia ${ }^{20}$ en Tosca, el rigor en Die Meistersingern von Nürnberg de Sixtus Beckmesser ${ }^{21}$, las veladas acusaciones de prevaricación que desliza el Gran Inquisidor ${ }^{22}$ de Don Carlo, el abogado Blind en Die Fledermaus ${ }^{23}$... El catálogo es tan rico como la provista bodega que descubre Raimbaud en Le Comte Ory.

Como iuslaboralista, debo reconocer que es difícil atraer el género a mi disciplina. El trabajo asalariado parece haber carecido de interés a los ojos de los libretistas, que en los tiempos en que la Cuestión Social se desarrollaba volvían sus ojos a escenarios lejanos en el tiempo o en la Geografía. Basta con considerar la obra de Puccini para encontrar que Il Tabarro es la única obra donde la situación de la clase trabajadora aparece reflejada, si bien sin intención social alguna. La ópera sería, como tantas veces se le ha reprochado, un arte burgués. En La bohème pueden ser pobres, pero artistas. La idea de trabajar no pasa por sus creativas mentes.

Podría pensarse que a medida que el siglo XX avanzaba el mundo del trabajo encontraría su acomodo sobre el escenario operístico. La olvidada I gioielli della Madonna de Wolf-Ferrari, casi contemporánea de Il Tabarro, refleja en parte este mundo, pero su argumento, una vez más, discurre por vías truculentas. Ni siquiera Dimitri Shostakovich llegó a acercarse al tema como sería debido, quizá porque le iba la vida en ello. Su Lady Macbeth del distrito de Mtsensk, apaleada por el 
propio Stalin en Pravda de acuerdo con la leyenda, podría haber abordado las relaciones de clase, pero el drama sexual-rural acabó imponiéndose.

La zarzuela, y en más concreto el género chico, ofrece en contraste un campo más abonado para reflejar el contexto histórico del nacimiento del Derecho del Trabajo, en la clásica lección primera donde se aborda este contenido. Junto al excelente documental Una mirada al trabajo, preparado para el centenario de la Inspección de Trabajo, la proyección y comentario de un fragmento, durante cinco breves minutos, puede tener gran resultado.

En efecto, el carácter más popular y menos elitista del género llevó a que los libretos de su tiempo recogieran menciones a los conflictos contemporáneos. Nada de trovadores, sino madrileños tabernarios. Al retratar ambientes contemporáneos, normalmente en Madrid mismo, era fácil para los autores deslizar observaciones sobre lo que estaba pasando, aun disfrazadas de chascarrillo. El tango de Wamba en El bateo, con música de Federico Chueca y libreto de Antonio Domínguez y Antonio Paso, contiene, probablemente, la descripción más gráfica que jamás se haya hecho de la lucha de clases en todo el género lírico: "Estamos de tal manera / que si esto siguiera así / la dinamita y el fuego / tendrán que venir / (...) / Pues tanto nos van haciendo / que al fin habrá que gritar / arriba los socialistas y abajo... / (...) / No se puede hablar". Zarzuela y cuestión social ofrecen, a mi juicio, un fértil campo de investigación que encajaría a la perfección en el tema histórico que abre la disciplina y permitiría introducir ejemplos musicales de lo que es, para bien o para mal, el género musical escénico español por excelencia.

Ya en el campo del Derecho material vigente, hay que traer a escena el dúo de Ascensión y Joaquín, Hace tiempo que vengo al taller, de La del manojo de rosas, de Pablo Sorozábal. Es difícil caracterizar mejor el despido disciplinario por "la disminución continuada y voluntaria en el rendimiento de trabajo normal o pactado" que con las palabras de ese intercambio lírico: "Cuando tengo una cosa que hacer, no sé lo que hago / Pues le veo cesante, por zumbón y por vago". De esta manera, la zarzuela encuentra también su acomodo en el programa de mano de esta propuesta, compartida con los estudiantes al iniciar el tema correspondiente a despido.

\section{3. ÓPERA Y DERECHO PENAL}

Dentro del panorama presentado, poca duda cabe de que el Derecho Penal es el ámbito donde la ópera campa a sus anchas. Si se esbozara una división entre las óperas en las que se cometen delitos y las que no, la parte del león sería para el primer conjunto. Este, a su vez se compondría de óperas trágicas y de óperas cómicas, pues incluso el crimen puede conducir al humor.

No cabe ser exhaustivo en este apartado, que necesitaría de por sí solo una monografía, que espero abordar algún día. Pero sí que pueden ponerse en conexión algunos de los delitos recogidos en el Código Penal con memorables escenas operísticas, que funcionarían como una nota al pie de página de la enseñanza jurídica, al inicio o al final de cada lección.

Por fuerza, el catálogo ha de iniciarse por los delitos contra la vida, el más abundante de los crímenes operísticos. Ruth Bader Ginsburg consideraba que Don Giovanni era una de las óperas más perfectas jamás escritas ${ }^{24}$. El debate se iniciaría con la muerte del Comendador a manos del 
seductor protagonista para reflejar este elemento de nuestro catálogo leporelliano de delitos, precedido de la agresión sexual de la que ha sido objeto Donna Anna. ¿Cuál es la calificación penal que procede, homicidio o asesinato, en el duelo desigual y nocturno entre un anciano desconcertado y un hombre enmascarado?

El asesinato abunda en los escenarios operísticos. Si consideramos la alevosía, ¿de qué otra manera puede calificarse la circunstancia de la muerte del rey Duncan en el Macbeth de Verdi? Un crimen notable, en el que es el guerrero escocés el que empuña el puñal, pero en el que la fascinante Lady puede ser considerada la inductora, la cooperadora necesaria, la encubridora de aquel corazón tan blanco ¿Hay mejor manera de empezar el estudio del Título I del Libro I del Código Penal?

"Precio, recompensa o promesa" hacen aparición también en la propia Macbeth, con la muerte de Banquo. Pero la atención ha de derivar hacia el tercer acto de Rigoletto del propio Verdi, si bien la muerte de Gilda, en el marco de una de las más maravillosas tormentas jamás compuestas, merece entrar más bien en el terreno de la alevosía. ¿Qué se podría decir a propósito del ensañamiento? ¿Es la diva Floria Tosca rea de asesinato o por el contrario ha actuado en defensa propia al apuñalar al barón Scarpia cuando este pretende agredirla sexualmente? ¿Se ensaña Ramfis en Aida al sepultar vivo a Radamés bajo la piedra fatal?

El homicidio imprudente viene de la mano de don Alvaro en La forza del destino, el delito de rapto lo perpetran los cortesanos del Duque en Rigoletto, el parricidio llega de la mano de Orestes en Elektra, el Dulcamara de L'Elisir d'Amore no es más que un vil estafador, el argumento de $L a$ Gazza Ladra gira en torno a un robo no cometido por la soprano... Sobran los ejemplos.

Para no ser acusados de reaccionarios en la elección de óperas, conviene otorgar un espacio al Billy Budd de Benjamin Britten, lo que permite presentar también a Herman Melville como algo más que el autor de Moby Dick. En esta ópera, cuyo reparto es íntegramente masculino, el juicio de un marinero en tiempo de guerra y su condena a muerte en aplicación de la ley marcial es el hilo conductor de la trama.

El catálogo relativo al Derecho Penal debe terminar, por fuerza, con el catálogo de las penas. El coro de prisioneros de Fidelio, una ópera sobre el abuso de poder y la prisión injusta en la ola de la Ilustración, debe tener un lugar de honor, siendo el acompañamiento perfecto para presentar las teorías del Marqués de Beccaria. No faltan las penas de deportación, como es la situación del crudo último acto de Manon Lescaut, donde la protagonista, demasiado libre en materia sexual a los ojos de su tiempo, es expulsada de Francia y enviada a las penurias de la salvaje Luisiana. En todo caso, la pena más habitual en el mundo operístico es la de muerte, ejecutada con todo aparato, como en Tosca o en el glorioso final de Andrea Chenier de Giordano, de forma un tanto arbitraria al estilo del Gran Inquisidor, o piadosamente fuera de escena, como es el caso de Manrico en Il Trovatore.

Dentro del repertorio operístico, no obstante, hay una ejecución particularmente conmovedora. La muerte de las Carmelitas de Compiègne, final de Dialogues de Carmélites, ilustra como pocas piezas musicales el horror del Estado desencadenado. La escena es la Revolución Francesa, pero el terror es el del siglo XX, el siglo del totalitarismo, el de los grandes cementerios bajo la Luna. Y es bueno que los estudiantes reflexionen sobre esas cosas. 


\section{LA VIOLENCIA DE GÉNERO, OBJETO DEL ARTE}

Es indiscutible, al abordar los aspectos jurídicos de las tramas operísticas, percibir el cambio de percepción en la sociedad de la violencia de género. Frente al repudio general con el que es recibida en nuestros días, el análisis de su tratamiento en las óperas de los siglos XVIII y XIX revela una normalización casi absoluta de los malos tratos. Solo el siglo XX ofrece tratamientos más actuales de esta cuestión, si bien los ejemplos son muy limitados.

La más grande de las paradojas operístico-jurídicas, en mi opinión, es la prodigiosa belleza con la que se ha plasmado esta aborrecible situación. Aunque óperas como La serva padrona, Le nozze di Figaro o Falstaff hagan vencedora a la mujer por su superior astucia, lo cierto es que la ópera es un género patriarcal, hija de su tiempo más incluso que de sus autores. De la misma manera que la pintura plasmó sin pudor los delitos sexuales de la mitología griega para representar el cuerpo femenino desnudo, las óperas recogen el sentir de su tiempo.

Un buen ejemplo de ello es el fallido intento de seducción de Corinna por parte del Cavaliere Belfiore en Il Viaggio a Reims. A los estudiantes no ha de resultarles desconocida la polémica a propósito del consentimiento en las relaciones sexuales, plasmada en el conocido lema "no es no". Primero se les hará escuchar la belleza sublime del dúo. Y después se comentarán con ellos las palabras del personaje; el "no" de una mujer ha de ser entendido en sentido contrario, porque nunca es sincero.

No podemos pasar por alto las circunstancias de la composición y el estreno de esta ópera, nada menos que con motivo de la coronación de Carlos X, con un libreto tan fastuosamente ultra que hace de Chateaubriand un liberalote y nada menos que Giuditta Pasta y Domenico Donzelli creando las partes. Pues bien, en ese regio escenario, el tenor expresa un argumento sobre la negativa de las mujeres que, por desgracia, sigue calando hoy entre la sociedad ${ }^{25}$. El problema, la paradoja antes mencionada, surge de la prodigiosa belleza con la que Rossini dotó musicalmente a estas líneas y la furiosa negativa de Corinna.

Con el paso del tiempo, la actitud de sociedad y compositores se irá modificando. Pero la historia de La Sonnambula de Bellini sigue siendo la de un celoso patológico a quien es difícil augurar una existencia feliz junto a Amina, aunque su rabia se exprese con la más hermosa de las melodías bellinianas en Tutto è sciolto. ¿Y qué decir del Batti batti, o bel Massetto de Don Giovanni? Con una música que es pura seducción, Zerlina no dice otra cosa que palabras que encontraríamos repulsivas en un reguetón ${ }^{26}$.

El Verismo, por su parte, plasmó este tema de una forma mucho más cruda y actual. El examen de la producción operística del siglo XX, que por falta de espacio no se abordará aquí, podría ofrecer un interesante resultado. Las conductas de los protagonistas en, por ejemplo, Cavalleria Rusticana, Pagliacci o Il Tabarro son presentadas bajo una clara óptica de denuncia. La violencia machista, con resultado de muerte incluso en dos casos, es repudiada por los autores. 


\section{ROMPIENDO LA CUARTA PARED: LOS PROBLEMAS EN EL TRABAJO EN LA ÓPERA}

Leoncavallo, en el Prologo de Pagliacci antes mencionado, invitaba a los espectadores a atender a los sufrimientos de los cantantes y no a sus pobres máscaras de histriones. De la misma manera, el análisis puede reconducirse desde los argumentos de las óperas a las condiciones reales en las que estas se realizan. Desde el punto de vista del Derecho del Trabajo, aquí surge la oportunidad de incorporar la ópera a la pedagogía ciudadana que se persigue. Las relaciones laborales en el mundo de la música clásica, a las que espero dedicar pronto mi tarea investigadora, son un campo fecundo de análisis.

En el campo de la discriminación, por ejemplo, es menester mencionar el caso de Deborah Voigt. La carrera de esta soprano estadounidense, de voz espléndida, está marcada por dos episodios susceptibles de ser caracterizados como discriminación derivada de la discapacidad, encajando en ella la obesidad, tal y como ha hecho el Tribunal de Justicia en los conocidos casos Kaltoft ${ }^{27}$ y Ruiz Conejero ${ }^{28}$.

En los primeros años de su carrera, audicionó para aquel titán de la batuta que fue Sir Georg Solti ${ }^{29}$, con la intención de grabar su primera Isolda. La valoración del húngaro transformado en británico fue atroz. Tras alabar la belleza de su voz, indiscutible, le preguntó con toda naturalidad “¿Por qué estás tan gorda?”. Voigt llegó a superar los 150 kilogramos, encarnando para el público general ese estúpido tópico sobre la corpulencia de las sopranos que supone ignorar que las cantantes de ópera fueron, durante siglos, la encarnación de la belleza, la clase y el glamour ${ }^{30}$. No en vano Irene Adler, la Mujer, tiene como profesión la de contralto, encarnando con particular éxito al paje Urbain en Les Huguenots.

El episodio más conocido, no obstante, en la trayectoria de Voigt fue su rechazo por parte del director de escena Peter Mario Katona para protagonizar Ariadne auf Naxos, de Richard Strauss, en la Royal Opera House de Londres. El regista había decidido que su protagonista debía vestir una petite robe noire y no cualquier otra ropa en la que Deborah Voigt pudiera cantar este papel en una ópera en la que, por cierto, Ruth Bader Ginsburg ${ }^{31}$ y Antonin Scalia hicieron un cameo en su representación en el Kennedy Center de Washington. Voigt fue sustituida por la más esbelta Anne Schwanewilms. ¿Discriminación o exigencia profesional? El debate con los estudiantes está servido.

No escasean las huelgas en el ámbito operístico. El autor de este trabajo se quedó sin ver Les Vêpres Siciliennes en la Ópera de París por el muy francés ejercicio de este derecho fundamental. También con Verdi de por medio, el caso más extraordinario de esquirolaje interno tuvo lugar en la representación de La Traviata en la Scala de Milán el 2 de junio de 1995. Ante la huelga de los profesores de la orquesta, el implacable Riccardo Muti hizo colocar un piano de cola en el centro del escenario. Con todo el reparto perfectamente caracterizado, incluyendo a aquella estrella fugaz que fue Tiziana Fabbricini, el director de Ravena procedió a tocar al piano la partitura de la ópera, acompañando a sus cantantes a lo largo de toda la ópera verdiana. 
Pero no es necesario salir de España para abordar casos de esquirolaje artístico similares. En noviembre de 2017, la Orquesta Sinfónica de Euskadi fue a la huelga durante las representaciones de Don Pasquale en el Teatro Euskalduna. El escenario pone de manifiesto lo complejo de las relaciones laborales en el mundo de la ópera, puesto que los profesores de la OSE son trabajadores de esta, tocando en funciones organizadas por la ABAO, la Asociación Bilbaína de Amigos de la Ópera. ¿Quién es el empresario aquí?

La ABAO no era el empleador atendiendo al parámetro salarial, aunque cabría preguntarse si estamos ante un supuesto de cesión de trabajadores si se considera el poder de dirección. La huelga era un conflicto laboral entre músicos y dirección, y a juicio de ABAO nada impedía recurrir a la sustitución de la orquesta: "se decidió trabajar la posibilidad de contratar una nueva orquesta. Esta alternativa ha resultado inviable debido al escaso tiempo del que se ha dispuesto, y por la imposibilidad de encontrar una orquesta de calidad que estuviese disponible para participar en las funciones". ¿Hubo una pretensión de esquirolaje interno? La cuestión se resolvió alla Muti, con el director de orquesta al piano.

Por otra parte, el verbo "protestar" tiene un significado propio en el mundo de la ópera. Más allá de lo que puede gritar el gallinero, en especial el loggione de la Scala, se conoce, de forma un tanto rancia, con este italianismo la práctica por la cual un teatro puede resolver de forma unilateral el contrato que ha celebrado con un cantante si considera que no está capacitado para interpretar con solvencia su papel. Sería así una facultad paralela al periodo de prueba, concediendo al empleador esa capacidad tremenda de resolución unilateral. Nótese que la base de la extinción es altamente subjetiva y puede sustanciarse en que el cantante no sea del agrado del director musical o del de escena.

Esta práctica sigue vigente hoy en día, como demuestra la sentencia de la Sala de lo Social del Tribunal Superior de Justicia del País Vasco de 20 de febrero de 2018². Contratada para cantar el papel de Donna Elvira, una conocida cantante española despertó, desde el primer día de ensayos, las reticencias de la directora musical del Don Giovanni que se había de representar en la programación de la ABAO. Conforme a la cláusula séptima de su contrato, recogida en los hechos probados, "en caso de que el artista sea protestado por el Maestro Director, y previa consulta a la Dirección de la ABAO, el artista percibirá únicamente una cantidad igual a la aportación para los gastos de viaje y/o alojamiento, convenida en la estipulación decimoquinta de este Contrato, quedando rescindido el mismo sin obligación de la ABAO de abonar ninguna cantidad más".

La recusación fue gestionada de forma discreta ante los medios de comunicación, si bien fue impugnada judicialmente. Desestimada la demanda de despido, la cantante recurrió en suplicación. De acuerdo con la sentencia, "ciertamente esa cláusula de protesta o recusación puede tener su validez en otros ordenamientos jurídicos, como el italiano, tal y como se indica en la sentencia recurrida, que también cita autor, dentro de nuestra doctrina científica, a favor de su validez y eficacia en nuestro ordenamiento jurídico. Pero nosotros no alcanzamos esta conclusión". La sentencia contiene otros aspectos de interés que, una vez más, no serán abordados aquí, pero que pueden generar un muy interesante caso práctico en el tema dedicado a la extinción del contrato de trabajo. 


\section{FINALE. ÓPERA Y MAFIA}

La asignatura de Derecho Penal tiene una relación privilegiada con la Cultura, no cabe duda. En ella es fácil transitar por los dominios de las distintas musas e incluso combinarlos. De ahí que sea especialmente feliz en dicha disciplina la puesta en práctica de todo lo que he propuesto. La presencia del crimen en el cine da todo tipo de facilidades que también se producen en el campo operístico.

No espere el lector encontrar, en esta parte final, una diatriba sobre los grupúsculos que dominan el mundo de la ópera, sobre los intereses económicos que mueven los hilos, sobre el modelo de negocio que impera en la lírica. Si quiere leer sobre eso, diríjase a los escritos de Norman Lebrecht. Tampoco será el momento de comentar el famoso montaje de Rigoletto de Jonathan Miller, en el que trasladaba, precursor, la acción de Mantua a Little Italy. No. Lo que va a encontrarse aquí son unas breves notas sobre la curiosa conexión entre la ópera y la mafia en el séptimo arte, en el cine.

Esta relación aparece en las dos modalidades artísticas aristotélicas, tanto en la comedia como en el drama. Basta con recordar a "Botines" Colombo y sus secuaces, en Con faldas y a lo loco, haciéndose pasar por amigos de la ópera italiana y usando precisamente Rigoletto como coartada para eximirse del terrible crimen que crea el hilo argumental de la película de Billy Wilder.

En otras ocasiones, la ópera no es parte de la trama, sino una presencia en la banda sonora. Continuando en lo ligero, Una terapia peligrosa utiliza en su banda sonora tanto La donna è mobile como el M'apparì de Martha. Otro hito del crimen televisivo, Los Soprano, es menos fecundo de lo que cabría esperar, pero incorpora fragmentos de Bajazet de Vivaldi y de La Rondine de Puccini en su soporte musical. Y el Intermezzo de Cavalleria Rusticana, por cierto.

Cualquier amante del cine sabe que El Padrino III no está a la altura de sus dos predecesoras. Pero en lo que aquí concierne, es el título perfecto: acercar a los estudiantes a través de un formato que les resulta familiar al mundo operístico.

Alejándose de la vida de crimen organizado de su padre, Anthony Vito Corleone decide abandonar el estudio del Derecho para dedicarse, nada menos, que a ser tenor. Obviamente, a Michael Corleone esto no le hace ninguna gracia e intenta imponer a su hijo la misma condición que los padres de Tito Gobbi y Alfredo Kraus impusieron a los suyos: acaba la carrera (Derecho y Peritaje Industrial, respectivamente). El díscolo bambino sigue adelante con sus propósitos, incitado por su madre, y la trama conduce a su debut operístico.

No cabe andarse con contemplaciones: es absolutamente inverosímil que un tenor novato vaya a tener como primer papel el Turiddu de Cavalleria Rusticana nada menos que en el Teatro Massimo de Palermo. Por no mencionar el hecho de que la gente entra y sale de la sala, nadie se entera del jolgorio criminal que tiene lugar, los curas llegan tarde... Asumamos, pues, la suspensión de la incredulidad que debería guiar siempre nuestros pasos en un teatro de ópera y pasemos por alto esta situación, por necesidades del guion. Ciertamente, no hay nada más intrínsecamente siciliano que ese título; sólo falta alguien comiendo cannoli para rematar el cuadro. Por cierto, ya 
hemos tenido antes un primer guiño en la narración, cuando Vincent Mancini muerde la oreja de su enemigo, Joey Zasa.

Nuestra función tiene lugar el 14 de abril de 1979, que no es el día en que murió Juan Pablo I. Momentáneamente ocultado por el asesino Mosca, podemos ver el cartel donde figuran los nombres de cuatro de los cantantes, todos ellos falsos. Franc d'Ambrosio aporta físico y voz a Anthony Corleone, antes de embarcarse en una exitosa carrera como el protagonista de $\mathrm{El}$ fantasma de la ópera. La verdadera voz operística es la de Paolo Gavanelli, que encarna vocalmente a Alfio, mientras escénicamente es Angelo Romero el que le da cuerpo. La dirección de escena, clásica excepto en la casi delirante procesión, corrió a cargo de Giuseppe di Tommasi. Como director de la orquesta, en otro de sus momentos de nepotismo habituales, Coppola escogió a su tío Anton. No hay que pasar por alto, además, que los títulos de crédito contienen un agradecimiento especial a Franco Zefirelli.

Obviamente, no toda Cavalleria Rusticana suena en El Padrino III. Su preludio nos acompaña al entrar en el Teatro Massimo y así la ópera arranca directamente con la Siciliana, para la cual Anthony necesita valerse de la partitura... Encadenamos directamente con la entrada de Alfio, mientras Al Neri y su caja de surtido Cuétara viajan hacia el Vaticano, y vemos fugazmente a Lola pasearse por escena, antes de que Turiddu entone su canto al vino. Por cierto, el único que parece conocer la ópera es don Altobello, que agita furiosamente las manos, como esta ópera merece para un temperamental siciliano.

Viene entonces el salto atrás a la procesión, que aparece postergada a su aparición en la ópera. Coppola se recrea aquí en una evocación del tenebrismo siciliano de Semana Santa, con la aparición de encapuchados, trasuntos de la muerte, un conmovedor velo negro que cubre el crucifijo y la aparición final del Redentor resucitado.

Lo último que veremos es el propio final de la ópera, cómo Turiddu termina el Addio, antes de que oigamos las fatales líneas de cierre. Y aquí entra el juego de los espejos. En la tremenda escena de las escaleras, donde el maravilloso Intermezzo suena bañando de paz y belleza la tragedia familiar, encontramos la desgarrada repetición de esas líneas, ahora ya dedicadas a Mary; vemos a Connie cubrirse la cabeza con un velo negro, de la misma manera en que lo había hecho Santuzza; y por último vemos el final reflejado, el padre que sostiene a su hija muerta.

El Padrino III es una declaración de amor, en definitiva, de Coppola por la obra de Mascagni. La demostración de que un genio, incluso cuando no está en su mejor momento, puede producir momentos dorados. Y la afirmación de la fuerza dramática de Cavalleria Rusticana, imbricada en el tapiz de la narración de la saga de los Corleone como un hilo del más valioso oro.

Podría contar mucho más sobre Pagliacci y los mafiosos. Pero el espacio concedido se termina. Recuerde el lector que Robert de Niro, en la piel de Al Capone, va a verla en Los Intocables de Elliot Ness y llora mucho con Vesti la giubba. Pero como dijo Kipling, eso es ya otra historia. 


\section{Bibliografía}

Abel Souto, M. (2011). Desde la literatura, ópera, cine y televisión hasta las ciencias jurídicas y el derecho penal mediante la nueva técnica pedagógica del aprendizaje basado en problemas. Dereito: Revista Xurídica da Universidade de Santiago de Compostela, 20,2.

Alonso Olea, M. (1992). Entre Don Quijote y Sancho, ¿relación laboral?. Madrid: Servicio de Publicaciones de la Universidad Complutense de Madrid.

Coderch, S. (2020). Gianni Schicchi y los notarios en la ópera, Scherzo. 359

Forlenza, F. (2003). Il diritto penale nella Divina Commedia: le radici del sorvegliare e punire nell'Occidente. Roma: Armando Editore.

Gil Otero, L. (2019). Innovación docente y capacidad de interconexión: especial mención al Derecho del Trabajo. En Investigación y docencia en Derecho: nuevas perspectivas. Madrid: Colex, Madrid.

Ginsburg, R. B. (2016). My Own Words. New York: Simon \& Schuster.

Littlejohn, D. (1992). The Ultimate Art. Essays Around and About Opera. Berkeley: University of California Press.

Miranda Boto, JM. (2009). La lectura de clásicos como refuerzo de la enseñanza del derecho: a propósito de "Marianela", de Benito Pérez Galdós. Dereito: Revista xuridica da Universidade de Santiago de Compostela, 18, 2.

Miranda Boto, JM. (2017). El Derecho en Tolkien. Madrid: Ediciones Cinca.

Pérez Adrián, E. (2000). Strauss. Discografía recomendada. Obra completa comentada. Barcelona: Península, Barcelona.

Rodríguez Álvarez, A. (2017). El Derecho Procesal en el arte, el cine, la literatura y la música: una primera lección para neófitos. ISLL Papers, 10.

Shippey, T. (2000). J.R.R. Tolkien. Author of the Century. Londres: Harper Collins.

NOTAS

${ }^{1}$ Una primera versión de este texto fue presentada como ponencia en el Congreso Internacional "Docencia universitaria, Dereito e Artes", celebrado en Santiago de Compostela en noviembre de 2020. Agradezco al Comité organizador la gentil invitación para hablar de lo que me apasiona y a Arturo Reverter, Victoria Stapells y María Pardal la lectura del primer manuscrito y sus observaciones. Debo dejar constancia también de mi agradecimiento a los revisores anónimos del texto, que aportaron las que son, muy probablemente, las mejores observaciones que ha recibido nunca un texto mío sometido a evaluación.

2 https://www.juntadeandalucia.es/export/drupaljda/WEB_Universitario_Andaluz.pdf. En el gráfico de su p.185 se recoge esta afirmación a propósito de la mayor parte del área de Humanidades: "suspender cautelarmente títulos e informar bien al alumnado".

${ }^{3}$ Me parece obligado reproducir aquí las inteligentes observaciones de uno de mis revisores anónimos: “¿Deberían introducirse referencias al fútbol, como fenómeno cultural que es? ¿Y si se trata de un deporte con cierta mala prensa como el pugilismo, a pesar de ser el deporte que más relacionado está con el cine, por ejemplo? ¿Y los toros? ¿El arte urbano a través de graffitis hechos en lugares públicos? ¿Serían cultura las técnicas y campañas de marketing —piénsese 
en el anuncio de Pepsi en el que se ofrecía un caza por siete millones de «Pepsi-puntos», y que un consumidor consideró una oferta vinculante susceptible de aceptación, solicitando la entrega del avión-?"

4 Y algunas de las más oscuras, como Dietrich Schwanitz.

5 "Conocer lo mejor que ha sido dicho y pensado en el mundo".

${ }^{6}$ Sobre este aspecto, me remito a mi propio trabajo, MIRANDA BOTO, 2009, p.313 y ss. Sugiero también la lectura de GIL OTERO, 2019, p.133 y ss.

7 "Cierto día se difundió por la Fábrica siniestro rumor: Rita de la Riberilla, una operaria, había sido cogida con tabaco. (... ) Cuando llevaron a la culpable al despacho del jefe, lo primero que hizo fue llorar sin responder; y al cabo, hostigada ya, aseteada a preguntas, se resolvía a confesar que «el marido» la abría a golpes si no le llevaba todos los días tres cigarros de a cuarto".

8 "Investiguemos ahora qué se necesita para tener trabajo, y veremos que son indispensables estas condiciones: 1. a Que $^{\mathrm{a}}$ Q haya medios de adquirir el instrumento de trabajo y de pagar al trabajador, o que él los tenga, si trabaja por su cuenta".

${ }_{9}$ MIRANDA BOTO, 2017, p.126 y ss.

${ }^{10}$ ALONSO OLEA, 1992.

${ }^{11}$ SHIPPEY, 2000.

${ }^{12}$ LITTLEJOHN, 1992.

${ }^{13}$ Dos propuestas integrales en ABEL SOUTO, 2011, p.183 y ss. y RODRíGUEZ ÁLVAREZ, 2017, pp. 1-14.

${ }^{14}$ Sobre las vicisitudes del libreto, PÉREZ ADRIÁN, 2000, p. 208 y ss.

15 "Bohnen essen, dann Schöpsenfleisch essen, nicht husten, seinen Hauptmann rasieren, dazwischen die fixe Idee pflegen!” ("Coma habas, luego coma carne de olla, no tosa, afeite a su capitán, ;atención a la idea fija en el medio!")

${ }^{16}$ FORLENZA, 2003.

17 “En el nombre de Dios, en el año de la Encarnación salvadora de Nuestro Señor Jesucristo de mil doscientos noventa y nueve, el día primero de septiembre, en la hora oncena, yo, el notario Amancio de Nicolao, ciudadano de Florencia, por voluntad de Buoso Donati escribo este testamento, que anula, revoca y deja irrito cualquier otro testamento".

${ }^{18} \mathrm{El}$ testamento se encuentra accesible en la base de datos de la Italian Renaissance Document Site: http://irdsproject.org/doc/897/

${ }^{19}$ Le ha dedicado su atención a esta figura CODERCH, 2020, pp. 79-81.

20 "È forza che si adempia la legge" ("Es necesario que se cumpla ley").

21 "Hier wird nach den Regeln nur eingelassen" ("Aquí habrá de ser de acuerdo

con nuestras reglas!”).

22 "Ed io, l'inquisitor, io che levai sovente sopra orde vil di rei la mano mia possente, pei grandi di quaggiù, scordando la mia fè, tranquilli lascio andar un gran ribelle... e il Re" "“Y yo, el inquisidor, yo que muchas veces levanté mi poderosa mano sobre viles órdenes de los reyes, por los grandes de este mundo, olvidando mi fe, dejo ir en paz a un gran rebelde... y al Rey").

23 “Nein, mit solchen Advokaten ist verkauft man und verraten, da verliert man die Geduld!” (“No, con tales abogados uno se vende y se traiciona, ;se pierde la paciencia!"), en las indignadas palabras de su cliente Gabriel Einenstein.

${ }^{24}$ https://www.aarp.org/politics-society/history/info-2015/opera-a-love-supreme-for-bader-ginsburg.html

25 "Finto è il rigore, - lo so per prova; così far sogliono - le belle ognor. Tal resistenza - no, non è nuova, l'uso la chiede, - ed il decor. Oggi combattono, - domani cedono, e salvar credono - il loro onor" ("Vencido está el rigor... está probado que así lo suelen hacer... las bellas damas. Tal resistencia... no, no es nueva. La costumbre, la duda... y el decoro hoy luchan... mañana ceden y creen así salvar... su honor. Vencido está el rigor... está probado que así lo suele hacer... las bellas damas").

26 "Batti, batti, o bel Masetto, la tua povera Zerlina! Starò qui come agnellina le tue botte ad aspettar! Lascerò straziarmi il crine, lascerò cavarmi gli occhi, e le care tue manine lieta poi saprò baciar! Ah, lo vedo, non hai core! Pace, pace, o vita mia! In contenti ed allegria notte e dì vogliam passar!" ("Pega, pega, bello Masetto, a tu pobre Zerlina: aquí estaré cual corderita aguardando tus golpes. Dejaré que me arranques el pelo, dejaré que me saques los ojos, y tus queridas manitas contenta después sabré besar. ¡Ah!, iya lo veo, no serías capaz! Paz, paz, ¡oh, vida mía! En la dicha y la alegría pasaremos día y noche").

${ }^{27}$ ECLI:EU:C:2014:2463

${ }^{28} \mathrm{ECLI}: \mathrm{EU}: \mathrm{C}: 2018: 17$ 
${ }^{29}$ https://www.theguardian.com/music/2015/feb/17/deborah-voigt-soprano-book-call-me-debbie-addiction ${ }^{30}$ Y que hoy en día lo siguen siendo, como Kristine Opolais, Elina Garanca u Olga Peretyatko.

${ }^{31}$ Imprescindibles las reflexiones de una figura tan icónica como ella en GINSBURG, 2016, en especial el capítulo "Law and Lawyers in Opera".

32 ECLI:ES:TSJPV:2018:518 